\title{
Biologics for hidradenitis suppurativa: an update
}

\author{
Katarzyna Włodarek¹, Małgorzata Ponikowska1, Łukasz Matusiak¹ \& Jacek C \\ Szepietowski*,1 \\ ${ }^{1}$ Department of Dermatology, Venereology \& Allergology, Medical University, Wroclaw, Poland \\ *Author for correspondence: jacek.szepietowski@umed.wroc.pl
}

\begin{abstract}
Hidradenitis suppurativa (HS) is a chronic, inflammatory dermatosis characterized by an occurrence of nodules, abscesses, sinus tracks and scarring. Its pathogenesis is multifactorial and still not fully understood, therefore, current systemic therapies still remain a serious challenge. Increased levels of several proinflammatory cytokines have been reported in patients suffering from HS, therefore biologics appear as a new approach to therapy for this condition. Adalimumab is the only one internationally registered agent and should be considered first after the conventional therapies appear insufficient. The efficacy and safety profile of some preparations, like infliximab and etanercept was confirmed so far in randomized trials, but there are some new biologics which are still being evaluated and require more rigorous examination.
\end{abstract}

First draft submitted: 1 July 2017; Accepted for publication: 4 November 2018; Published online:

7 December 2018

Keywords: acne inversa $\bullet$ biologic drugs $\bullet$ biologics $\bullet$ biologic therapy $\bullet$ hidradenitis suppurativa $\bullet$ management $\bullet$ therapy • treatment

Hidradenitis suppurativa (HS), also known as acne inversa, is a chronic, recurrent, inflammatory dermatosis characterized by an occurrence of deep, painful lesions like nodules, suppurative abscesses, sinus tracks and scarring [1]. The disease occurs in body areas rich in apocrine glands, including mainly axillae, groin and anogenital region [2]. The changes often spread to the buttocks, the anal area or the woman's interbreast area [1]. The pathogenesis of HS is still not fully understood. The condition is multifactorial and probably results from a combination of genetic, hormonal (mainly hyperandrogenism) and environmental factors. Pilosebaceous unit occlusion, hyperkeratinization and bacterial superinfection are now considered the main pathogenetic mechanisms [2]. Tobacco smoking, drugs and obesity are recognized as the major risk factors for HS development. Moreover, a direct relationship with smoking and the severity of the disease symptoms has been proven [3,4]. The incidence of the disease is around $1 \%$ with a female predominance (female to male ratio of 3:1), however, there is also a report suggesting even a $4 \%$ prevalence [5,6]. HS has been associated with several comorbid disorders known as immune-mediated inflammatory diseases (IMIDs), such as Crohn's disease, colitis ulcerosa, seronegative arthritis and pyoderma gangrenosum [7,8].

HS is a debilitating disease resulting in patient mutilation, it is also associated with chronic pain sensation. It was documented that HS patients have a poor quality of life (QOL) directly correlated to the severity of the disease. QOL impairment occurs also more frequently than those found in some other dermatoses such as psoriasis, atopic dermatitis and chronic urticarial [9-12].

Due to the multifactorial pathogenesis of the disease, the treatment of HS often occurs as a therapeutic challenge. European S1 guideline for HS has been developed by a group of experts, but an unambiguous treatment algorithm has not been established. Thus, the preparations most commonly used in HS therapy include $1 \%$ topical clindamycin, systemic antibiotics (including tetracycline or clindamycin and rifampicin combination), retinoids and hormone therapy [13]. It is important to emphasize that pharmacological therapies should be introduced while treating HS as early as possible, in order to avoid complications such as scars, sinus tracts or malignancies development (Marjolin's ulcer occuring in previously traumatized and chronically inflamed skin areas) [14].

Biologics that have been used for almost 20 years for the treatment of IMIDs, have also proven to be a promising therapeutic option for HS sufferers. They are successfully used in patients with moderate to severe HS when the conventional systemic therapies proved insufficient. 


\section{TNF- $\alpha$ inhibitors}

TNF- $\alpha$ is a cell signalling protein (cytokine) produced by many cell types such as activated macrophages, mast cells, $\mathrm{CD}^{+}$lymphocytes, NK cells and neutrophils. It plays a key role in the inflammatory response in humans and is involved in various inflammatory responses, including acute phase reaction. TNF- $\alpha$ is generated as a precursor form called tmTNF (transmembrane). Its biological activity is associated with binding two receptors, TNFR1 (found in most tissues) and TNFR2 (expressed typically in cells of the immune system). The cytokine acts by promoting an expression of adhesion molecules, neutrophils migration and phagocytosis of macrophages. It also stimulates production of a number of mediators, including CRP, IL-1 oxidants and the inflammatory lipid PGE , $^{2}$ as well as activates caspases, intracellular signaling NF-kB and MAPK. Currently, TNF- $\alpha$ is believed also to play an important role in the pathogenesis of HS lesions. This is indirectly confirmed by the fact that TNF- $\alpha$ inhibitors have been successfully used in HS therapy for several years.

The relationship between TNF- $\alpha$ blockade and HS improvement was first noticed in 2001, when Martinez et al. [15] observed an improvement in the condition of skin lesions in the course of HS among patients undergoing anti-TNF- $\alpha$ therapy due to co-morbid Crohn's disease. This observation subsequently prompted other researchers to do a deeper analysis of this issue to characterize the proinflammatory and anti-inflammatory cytokines profile in the body of patients suffering from HS.

Matusiak et al. [16] showed a significantly elevated level of TNF- $\alpha$ in the blood of HS patients compared with control, whereas the level of cytokine did not correlate with the disease severity, its duration or BMI. In another study, Van der Zee et al. [17]. reported increased expression of proinflammatory TNF- $\alpha$ and IL-1 $\beta$ as well as anti-inflammatory Il-10 in patients with HS compared with the healthy controls in both the diseased skin and perilesional area. It was also five-times higher than the values observed in psoriasis. Similar conclusions regarding the level of TNF- $\alpha$ in patients with HS have been reached by Mozeika et al. [18] showing elevated levels of cytokine in the skin, apocrine glands and hair follicles.

However, there are also opposite reports that have shown reduced levels of substances associated with innate immune response, including TNF- $\alpha$ in both the blood and tissues of patients suffering from HS. Van der Zee et al. [19] when assessing TNF- $\alpha$ levels in the skin of patients undergoing 16-week adalimumab therapy, did not observe any significant differences in cytokine concentration before and after treatment.

Despite contradictory reports, TNF- $\alpha$ seems to play a significant role in the pathogenesis of HS, the best confirmation of which are numerous positive reports on the use of TNF- $\alpha$ blockers in the therapy of this condition.

\section{Adalimumab}

Adalimumab (ADA) is a fully human monoclonal antibody against TNF- $\alpha$. It binds with a high specificity and affinity to soluble and membrane-bound TNF- $\alpha$ and blocks its biological activity. ADA regulates the innate immune response by affecting the levels of proinflammatory cytokines such as Il-6, Il-8, Il-1 $\beta$ and sTNF-RI [20]. Treatment with ADA was also associated with decreased number of inflammatory leukocyte subsets including monocytes, macrophages, dendritic cells, T-helper and B lymphocytes [21].

ADA is administered by subcutaneous injections, and its highest effectiveness, according to European guidelines, is obtained with a dose regimen of $40 \mathrm{mg}$ once weekly. There is no dose adjustment for patients with obesity [13]. The drug is contraindicated in NYHA class III-IV heart failure, history of tuberculosis or other severe infections, severe liver disease, demyelinating processes, malignancies, pregnancy or lactation. Women of childbearing age should therefore receive contraception up to 5 months after treatment [13].

The first reports of scientists about the efficacy of ADA on HS came from several case series. In the majority of studies, ADA was administered with the dosing regimen previously adopted for the treatment of psoriasis (80 $\mathrm{mg}$ at week $0,40 \mathrm{mg}$ at week 1 and then $40 \mathrm{mg}$ every other week) [22]. The effects were satisfactory in accordance to both efficacy and safety of the treatment [21,23-33]. In subsequent years, prospective studies based on larger groups and longer observation of patients were published.

Blanco et al. [34] perform a retrospective analysis of a group of six patients treated with ADA for refractory HS. The initial dosage was $40 \mathrm{mg}$ every other week and was increased to $40 \mathrm{mg}$ weekly if the condition was inadequately controlled. All patients reported a marked reduction in the number of affected areas of the body, nodules, fistulas and laboratory parameters, as well as an improvement in the Dermatology Life Quality Index (DLQI). The mean follow-up period in this study was 21.5 months. Arenbergowa et al. [35] used ADA in a group of eight patients with severe, recalcitrant HS (Hurley grade III). Patients were treated for 1 year with a standard psoriasis dosing regimen and after that monitored for 1 year. Clinically significant improvement was observed in all patients within 4-6 
weeks. Three of them remained stable with no relapse during the follow-up period. The average time to recurrence was 9.5 months.

In another open-label prospective study by Sotiriou et al. [36] 15 patients with moderate to severe HS were treated with ADA in a different from previously mentioned dose regimen: $80 \mathrm{mg}$ was administered at week 0 , and then $40 \mathrm{mg}$ weekly for 24 weeks. After this time, a significant decrease in Sartorius score was reported. DLQI, as well as disease activity evaluated by visual analogue scale (VAS), also showed a marked reduction at week 24 . There was however a significant worsening at week 48 , and recurrences after discontinuation of treatment were noticed after mean time of 11 weeks.

In the study by Amano et al. [37] the results were not so promising. Ten patients were enrolled in this study and administered ADA for 12 weeks at doses of $160 \mathrm{mg}$ at week $0,80 \mathrm{mg}$ at week 1 and $40 \mathrm{mg}$ every other week. At week 12, none of the patients were classified as a responder compared with the baseline. There was also no statistically significant improvement in pain and QOL.

The first randomized, double-blind, prospective, placebo-controlled trial evaluating the efficacy and safety of ADA in the treatment of HS was carried out by Miller et al. [38] 21 patients suffering from moderate to severe HS (Hurley stage II or III) for at least 6 months were randomized 1:2 (placebo: active treatment). Thus, 15 patients received $80 \mathrm{mg} \mathrm{ADA}$ at week 0 , followed by $40 \mathrm{mg}$ every other week for 12 weeks, while six patients received placebo. A marked reduction in Sartorius score occurred after 6 weeks and in the ADA group when compared with placebo control. However, no significant change in the Hurley score, VAS or DLQI was seen after 12 weeks.

Kimball et al. [39] conducted another larger, randomized, placebo-controlled two-phased study. It included a group of 154 patients with moderate and severe HS (HS Physician Global Assessment [HS-PGA] score of moderate or worse) who had previously reported intolerance or lack of response to oral antibiotics. During period 1 (blinded phase) patients were randomized in a 1:1:1 ratio to ADA 40 mg every week, ADA 40 mg every other week (EOW) and placebo for 16 weeks. Period 2 was open-label and all patients were treated with ADA $40 \mathrm{mg}$ EOW. At weeks 28 or 31 patients with a suboptimal response (HS-PGA score of moderate or worse) were switched to weekly dosing. At week 16, a significantly greater proportion of patients in the weekly group (17.6\%) achieved a clinical response (HS-PGA score of clear, minimal or mild with at least a 2-grade improvement relative to baseline) compared with patients in EOW (9.6\%) and placebo (3.9\%) groups. This group also achieved a significantly greater reduction of pain (assessed by using VAS questionnaire). However, after the switch from weekly to EOW dosing in period 2, a decrease in response was reported. These observations suggest that the most effective dosing regimen for ADA is $40 \mathrm{mg}$ every week. During the study, headaches and injection site reactions were the most frequently reported side effects. Serious adverse event rates in all three groups were: 7.8, 5.8 and 3.9\%, respectively, and the worsening of HS, infectious complications and anemia were the most common.

The most recent and most significant Phase III trial for the evaluation of the efficacy and safety of ADA in HS therapy is the PIONEER I and II [40]. These multicenter studies, in which 307 and 326 patients participated, respectively, were similarly designed with two double-blind and placebo-controlled periods. In period 1 (12-weeks), patients were randomized in a 1:1 ratio into two groups - one receiving ADA (160 mg at week $0,80 \mathrm{mg}$ at week 2 and $40 \mathrm{mg}$ weekly from week 4 through week 12), the second matching placebo. All patients who received ADA in period 1 and continued into period 2, were then re-randomized 1:1:1 to ADA $40 \mathrm{mg}$ weekly, every other week or placebo. Patients who were in the placebo group in period 1 were reassigned to ADA $40 \mathrm{mg}$ weekly (PIONEER I) or placebo (PIONEER II) for 24 weeks. Moreover, in PIONEER II an adjuvant therapy was also allowed (19\% of patients received concomitant oral antibiotics).

The primary efficacy end point was HiSCR (Hidradenitis Suppurativa Clinical Response) defined as more than $50 \%$ reduction in total abscess and inflammatory nodule count, and no increase in abscess and draining fistula count at week 12 comparing to a baseline.

At week 12, HiSCR achievement rate was significantly higher for patients in the ADA group compared with the placebo group ( 41.8 vs $26 \%$ in PIONEER I and 58.9 vs $27.6 \%$ in PIONEER II). The marked improvement was observed in ADA group as early as 2 weeks into therapy.

Moreover, in the PIONEER II, although not in PIONEER I, ADA proved to be significantly more effective than placebo in secondary outcomes including: pain reduction (measured with Patient's Global Assessment of Skin Pain), disease severity (in Modified Sartorius score and Hurley Stage), as well as the number and morphology of skin lesions. This group also had statistically significant improvement in quality of life (DLQI). Also in this study, under ADA treatment tolerance was satisfactory. The most commonly reported adverse effects were headaches and infections (especially upper respiratory tract and the urinary tract infections). During period 1, serious adverse 
events were observed in $1.3 \mathrm{ADA}$ versus $1.3 \%$ patients in placebo group (PIONEER I) and 3.7 versus 1.8\% respectively, in PIONEER II. By period 2, these rates were up to $4.6 \%$ with a similar frequency for both groups and studies.

ADA has therefore proved to be a drug with greater efficacy in HS treatment and a similar safety profile compared with a placebo.

In all of the studies, adverse side effects after administration of this preparation were usually mild to moderate. Relatively common adverse drug reactions were injection site reactions and infections, including serious infections such as pneumonia, arthritis, diverticulitis and pyelonephritis. Reactivations of latent tuberculosis or hepatitis B virus, as well as neurological and hematological complications were also reported during the course of a treatment [14]. Very rarely, malignancies (including lymphomas, squamous cell carcinoma or breast cancer) occurred [38,41]. A few cases of paradoxical reactions after ADA administration, due to other diseases therapy, were reported [42].

Therefore, ADA seems to give promising results both in effectiveness of HS therapy and safety of use. According to current evidence it also improves patients' QOL, reduces pain as well as depressive symptoms [43,44]. Three large prospective studies on ADA on a total estimated number of 914 patients are currently underway [45].

ADA is the only biologic drug approved by the US FDA and EMA for therapy of moderate to severe HS in adult patients after failure of classic treatment.

\section{Infliximab}

Infliximab (IFX) is a chimeric (mouse/human) monoclonal IgG1 class antibody that works against TNF- $\alpha$. Similarly, to ADA, it binds to both soluble and transmembrane receptor-bound TNF- $\alpha$ and neutralizes its proinflammatory activity.

IFX is administered by intravenous infusion at a dose of $5 \mathrm{mg} / \mathrm{kg}$ body weight at weeks: $0,2,6$ and then regularly in 8-week intervals for a long-term therapy [13]. Due to the possible infusion reactions, patients should remain under observation during the infusion and 1 hour after the drug administration [13].

A long-term prospective trial was carried out by Paradela et al. [46] on a group of ten patients suffering from moderate to severe refractory HS. IFX was administered intravenously in the previously mentioned dose regimen. Response, defined as more than 50\% decrease in HSS (hidradenitis suppurativa score) comparing with baseline was achieved in eight patients. However, disease recurrence was noticed in 4 patients after the mean period of 37 weeks.

In another prospective, interventional study by Lesage et al. [47] ten patients were treated with IFX $5 \mathrm{mg} / \mathrm{kg}$ at weeks 0, 2, 6 and then every 4 weeks. A significant decrease in disease severity (assessed in Hurley score) as well as QOL improvement was noted in all subjects. Complete efficacy (defined as the absence of HS flares) was obtained for two patients and partial efficacy (moderate flares with no need for surgery) for eight.

The only one randomized, double-blind, placebo-controlled study on the efficacy and safety of IFX in HS therapy was performed by Grant et al. [48] on a group of 38 patients.

In the first phase of 8-weeks duration, patients with moderate to severe HS (HS Severity Index score greater than 8) were randomized to treatment with IFX $5 \mathrm{mg} / \mathrm{kg}$ at weeks $0,2,6$ or matching placebo. The groups were then unblinded in the second phase of the study, and placebo patients had the opportunity to change therapy to the IFX for another 22 weeks. The last observational phase was followed until week 52. HSSI was used to assess the disease activity. At week 8 it was noted that significantly greater number of patients treated with the active drug achieved at least 50\% improvement in skin lesions compared with placebo. Interestingly, a similar effect was achieved by subjects that switched from placebo to IFX in the second phase of the study. Also reduction of DLQI, VAS, PGA (Physician Global Assessment) score and laboratory inflammation markers was significantly greater in IFX-treated patients comparing with placebo. The mean DLQI change from baseline for patients treated with IFX was 10.0 and in placebo group it was 1.6, and the mean VAS change was 39.0 and 0.6 , respectively.

Tolerance under IFX treatment was satisfactory, with, most commonly, mild adverse effects observed, including headaches, nausea and infections. Serious adverse events after IFX administration were similar to those reported during ADA therapy and mainly involved: reactivation of latent tuberculosis, Hepatitis B, hepatosplenic T cell lymphoma, hematological complications or neurologic events.

In the retrospective study conducted on two cohorts, each of ten patients, Van Rappard et al. [49] compared the effectiveness of IFX and ADA in HS therapy. Ten patients were treated with ADA $40 \mathrm{mg}$ every other week, and the second group with IFX $5 \mathrm{mg} / \mathrm{kg}$ at weeks $0-2-6$. A significant improvement in skin lesions, as well as reduction of the inflammatory laboratory parameters was obtained in both groups of subjects. The mean decrease 
Table 1. Level of evidence and response rate for studies on TNF- $\alpha$ inhibitors.

\begin{tabular}{|c|c|c|c|c|c|c|}
\hline \multirow[t]{2}{*}{ Biologics } & \multirow[t]{2}{*}{ No. of papers } & \multicolumn{3}{|c|}{ Level of evidence $^{\dagger}$} & \multirow[t]{2}{*}{ Responders $(\%)^{\ddagger}$} & \multirow[t]{2}{*}{ Nonresponders (\%) } \\
\hline & & A & B & C & & \\
\hline Total & $69^{\S}$ & 4 & 25 & 40 & $55,3 \%$ & $44,7 \%$ \\
\hline Adalimumab & 21 & 2 & 7 & 12 & $54 \%$ & $46 \%$ \\
\hline Infliximab & 38 & 1 & 12 & 25 & $82 \%$ & $18 \%$ \\
\hline Etanercept & 10 & 1 & 6 & 3 & $54 \%$ & $46 \%$ \\
\hline
\end{tabular}

in Sartorius score compared with baseline was 54\% in the IFX group and 66\% for the ADA group. However, only the improvement in the IFX group remained significant after one year of observation. Both preparations were beneficial, but IFX happened to be more effective in all aspects than ADA not only in decreasing disease severity, but also in improving QOL, normalizing of laboratory parameters and durability of achieved remission. No serious adverse effects were noticed in both groups.

\section{Etanercept}

Etanercept (ETA) is recombinant fusion protein that binds to transmembrane form of TNF- $\alpha$ and inhibits it. It is administrated by subcutaneous injections. It is approved by the US FDA for treatment of rheumatoid arthritis, plaque psoriasis, psoriatic arthritis, juvenile idiopathic arthritis and ankylosing spondylitis [50].

In a series of cases [51-53] as well as open cohort studies [54-59] conducted on groups of 4 to 15 patients who received ETA in doses of $25 \mathrm{mg}$ twice weekly or $50 \mathrm{mg}$ once/twice weekly, promising results regarding the efficacy of the drug in HS therapy were obtained.

Cusack et al. [56] reported a significant reduction in self-reported disease activity (mean reduction of 61\%) as well as an improvement in the QOL (mean reduction in DLQI scores of 64\%). In another study by GiamarellosBourboulis et al. [55] ETA was administered in a dose of $50 \mathrm{mg}$ once weekly for 12 weeks. More than $50 \%$ decrease of disease activity (according to the Sartorius scale) was reported in $6 / 10$ patients at week 12 and $7 / 10$ patients at week 24 . The reduction of VAS scores was noticed in 7/10 and 6/10 patients respectively.

Only one randomized, double-blind, placebo-controlled trial assessing the efficacy of ETA in moderate and severe HS has been published, but the results were unsatisfactory. It was carried out by Adams et al. [50] on a group of 20 patients. ETA $(50 \mathrm{mg})$ or placebo was administered twice a week for 12 weeks. After that, all subjects received open-label ETA in the same dose regimen for 12 more weeks. At 12 or 24 weeks, there was no significant difference in patient global assessment, physician global assessment or QOL (assessed with DLQI) between ETA and placebo groups.

In most of the published studies, ETA was well tolerated and the most commonly reported adverse reactions were injection site reactions and infections. However, in one patients bilateral Candida chorioretinitis followed by septicemia was described [52].

Reports differ as to the ETA efficacy in HS, which, in the light of the more confirmed efficacy of ADA and IFX, argues for the greater utility of these preparations in HS therapy. The dominance of the other two TNF- $\alpha$ inhibitors over ETA can be explained by the fact that these drugs bind to both soluble and transmembrane TNF- $\alpha$, whereas ETA inhibits only the transmembrane form [14].

Significantly increased expression of TNF- $\alpha$ found in HS sufferers supports the use of TNF- $\alpha$ inhibitors as a therapy of this condition. These preparations are also the most widely investigated biologic agents for the efficacy and safety of use. Both experimental and clinical trials have demonstrated the rationale behind using TNF- $\alpha$ inhibitors in HS treatment. The results of the published studies on ADA, IFX and ETA are summarized in the Table 1.

In total 69 papers were analysed, mostly including case reports and case series, but also randomized controlled trials for each preparation, which were mentioned above. The level of the study evidence was divided into three groups, which were respectively: A (randomized controlled trials), B (lower-quality clinical trials), C (case reports and case series). The patients were categorized as 'responders' or 'non-responders', according to the criteria established for each of the analyzed studies (e.g. decrease in HSSI, PGA score, achieving HiSCR). If no individual results were reported for each subject in a study, all patients were classified according to the mean achieved efficacy. Patients 
receiving placebo in the cohort studies were not included. The highest response rate was observed with IFX and the percentage of responders to this preparation was $82 \%$ compared with $54 \%$ for ADA and ETA. However, it is important to emphasize that the highest quality of evidence was identified for ADA and many more patients were analyzed after its administration than after IFX and ETA, which makes the result of ADA efficacy the most reliable. Smaller studies of IFX and ETA, in which nonvalidated measurements were used to assess the effectiveness of the therapy also do not determine high quality of evidence. Overall, the quality of evidence was much lower for IFX and ETA than for ADA and differed between the preparations, making it difficult to compare these agents directly. Therefore, larger randomized controlled trials are needed to precisely estimate the effectiveness of these preparations in HS therapy.

\section{Other biologics}

\section{Anakinra}

Anakinra (ANA) is a recombinant IL-1 receptor antagonist. It competitively blocks the binding of naturally occurring IL-1 (IL-1 $\alpha$ and IL-1 $\beta$ ) to its receptor and inhibits its biological activity. IL-1 (similarly to TNF- $\alpha$ ) is one of the major mediators of the inflammatory response that are also involved in the pathogenesis of HS [60].

Due to its immunomodulatory and anti-inflammatory properties, ANA may occur as a new, promising therapeutic approach in the management of HS and an alternative for patients who have failed to respond to other treatment regimens, including TNF- $\alpha$ blockers. ANA was originally registered for the treatment of moderate to severe rheumatoid arthritis. However, a successful off-label use of this drug was also reported in various conditions, often of autoimmune background, including psoriasis, atopic dermatitis, pyoderma gangrenosum, Schnitzler's syndrome, Sweet's syndrome or SAPHO syndrome [61]. In HS, ANA is typically administered by subcutaneous injections in a dose of $100 \mathrm{mg} /$ day, which corresponds to the dosage regimen for rheumatoid arthritis.

Different reports regarding to the efficacy of ANA in the management of HS occurred. In a prospective open-label study, Leslie et al. [62] assessed the effectiveness, safety and tolerability of ANA in HS management. The group of six patients with moderate to severe HS were treated with daily ANA $(100 \mathrm{mg} /$ day $)$ for 8 weeks, which was the active phase of the therapy, followed by 8 -weeks observation phase. Inclusion criteria for this study included minimum modified Sartorius score of 25 or greater and presence of active skin lesions in at least two anatomic areas of the body. As a result, all of the five patients who completed the study (one subject was lost to follow-up because of socioeconomic factors) achieved a clinically meaningful improvement after 8 weeks of therapy. A mean decrease of modified Sartorius score was 34.8 points. Patients' QOL was also improved, and the average decrease in the DLQI was -8.4 points, which is comparable with that obtained under ADA therapy in a study conducted by Kimball et al. [39]. However, relapse occurred in HS disease activity as well as others assessed parameters after an 8-week follow-up. ANA was well tolerated - no adverse events were reported in any of the study participants during the entire treatment nor the follow-up period.

A larger randomized double-blind, placebo-controlled clinical trial in a group of $20 \mathrm{HS}$ patients (Hurley stage II or III) was conducted by Tzanetakou et al. [63]. Patients were randomized in a 1:1 ratio, to receive placebo or ANA subcutaneously $100 \mathrm{mg}$ once daily for 12 weeks (treatment phase), and then remained under observation for the next 12 weeks. At the baseline visit, the patients were evaluated among the disease activity, number of skin lesions, affected areas and QOL. In addition, peripheral blood samples were also taken from the subjects and mononuclear cells were stimulated to cytokine production. The study confirmed ANA as a potentially efficacious management in HS therapy.

A total of $78 \%$ of patients treated with ANA achieved a good clinical response (decrease of disease activity score) after 12 weeks of active therapy comparing to $30 \%$ of the placebo group.

After 24 weeks, these results were 67 and 20\%, respectively, and the time to disease exacerbation was prolonged in patients treated with ANA. Moreover, there was also a decrease in the production of IFN- $\gamma$ in ANA group, and the production of interleukin 22 was increased. No serious adverse effects of the therapy were noticed.

Zarchi et al. [64] reported the case of a 37-year-old obese patient $(B M I=40)$ who was successfully treated with ANA $200 \mathrm{mg}$ daily, after the failure of other therapies, including IFX and ADA.

Despite promising reports confirming the efficacy of ANA in the treatment of moderate to severe HS, a few cases of 'nonresponse' to this treatment have also been reported [65-67].

Menis et al. [65] described even a worsening of skin lesions as well as DLQI and PGA in one of the two patients administered to with ANA. Due to contradictory reports in previously published studies, there is a need to assess the efficacy and safety of ANA in randomized trials with large groups of patients in the future. 


\section{Ustekinumab}

Ustekinumab (UST) is a human IgG1 class monoclonal antibody directed against the p40 subunit of IL-12 and IL-23, which regulates specific components of the immune system.

Both IL-12 and IL-23 are involved in differentiation and activation of Th cells subsets (Th1 and Th17 respectively) which release other proinflammatory cytokines [68]. Due to their mechanism of action, IL-12 and IL-23 play a role in the pathogenesis of IMIDs by dysregulation of the immune system, thus UST has been successfully used in the treatment of disorders such as psoriasis, Crohn's disease or HS [69]. Furthermore, Schlapbach et al. [70] reported an increased expression of IL-12 and IL-23 in lesional skin of HS sufferers, which was related to infiltration of papillary and reticular dermis by macrophages. These data provide a rationale for UST as a new therapeutic approach for HS therapy.

In a study by Gulliver et al. [71] three cases of patients were reviewed to assess the efficacy of UST therapy. All subjects were administered with UST by subcutaneous injections in a previously mentioned dose. Different outcome was achieved in each subject. Complete disease remission in one of the patients was obtained at month 6 , while $25-49 \%$ improvement was noticed in the second subject and no treatment effect in the third.

According to results of three other case reports, which were published by different authors [72-74], all patients reported a partial or complete response to UST therapy, however the effect of the treatment was not rapid and appeared within several months from the beginning of drug administration.

Blok and colleagues [75] conducted the only uncontrolled open-label clinical trial with prospective design to evaluate the efficacy of UST in HS therapy. 17 patients with moderate to severe HS (Hurley stage II-III) were included and treated with UST according to the further psoriasis dosing regimen: $45 \mathrm{mg}$ s.c. (increased to $90 \mathrm{mg}$ for patients weighing $>100 \mathrm{~kg}$ ) at week 0, 4, 16 and 28. Results were promising - moderate to marked improvement of skin lesions (according to modified Sartorius score) was achieved in $82 \%$ of patients and the HiSCR in $47 \%$ at week 40 . Moreover, $41 \%$ of subjects demonstrated clinically significant improvement in the DLQI. It was also noticed that the milder course of the disease and the lower leukotriene A4-hydrolase serum concentration were associated with a better response to UST therapy. The most commonly reported adverse events in this study were fatigue, headaches and upper respiratory tract infections.

Despite promising effects of UST in HS therapy, other preparations with better evidence for efficacy, such as ADA or IFX, should be considered first [76]. Regarding its unique mechanism of inhibiting IL-12/23, UST may provide a potential new therapeutic approach for HS in some patients after failure of other therapies.

\section{Secukinumab}

Secukinumab (SEC) is a fully human monoclonal antibody and is directed against IL-17A.

Recently published data confirmed that the level of IL-17A in the blood of HS patients is significantly elevated, compared with that found in healthy volunteers and directly correlates with the severity of the disease [77]. The expression of IL-17A was also enhanced in lesional as well as perilesional skin of HS sufferers [78]. IL-17A activates neutrophils and lymphocytes and induces the expression of proinflammatory cytokines including IL-1 $\beta$, IL- 6 and TNF- $\alpha$. SEC binds with a high selectivity to IL-17A and inhibits the inflammatory cascade [77].

Only three cases have been published on SEC in HS treatment after failure of multiple pharmacologic therapies, including biologics. The drug was administered as a subcutaneous injection at a dose of $300 \mathrm{mg}$ weekly (according to scheme 0-7-14-21-28) and then once a month as a maintenance therapy. In a study by Thorlacius et al. [79], the number of lesions reported by a patient was reduced from 23 to 7 and pain VAS from 5 to 3 at week 12 comparing to baseline. During the course of the treatment oral candidiasis occurred in the patient.

In the second case that was reported by Schuch $e t$ al. [80], a significant decrease in inflammatory nodules, as well as white blood cell count and CRP levels were observed. The patient did not experience any adverse effects related to the administered therapy. Jørgensen et al. [81] also reported a marked improvement in a patient treated with SEC, expressed by a remarkable reduction in VAS, DLQI, HSS and IHS4 (International Hidradenitis Suppurative Severity Score) after 6 months of therapy.

Currently, SEC is being tested in a randomized placebo-controlled trial in a group of $21 \mathrm{HS}$ patients who receive $300 \mathrm{mg}$ weekly for 4 weeks followed by $300 \mathrm{mg}$ every 4 weeks. Treatment efficacy will be assessed after 24 weeks and the only outcome in this study is achievement of HiSCR. Its results may be helpful in evaluating the therapeutic approach of targeting Il-17 in HS [82]. 
Table 2. Ongoing trials on other biologic agents in hidradenitis suppurativa treatment (situation as at 20 June 2018 )

\begin{tabular}{|lllll|}
\hline Drug & Mechanism of action & Phase of study & US NCT number & Study sponsor \\
\hline MABp1 & IL-1 $\alpha$ inhibitor & Phase II & NCT03512275 & XBiotech, Inc. \\
\hline CJM112 & IL-17A inhibitor & Phase II & NCT02421172 & Novartis Pharmaceuticals \\
\hline Bimekizumab & IL-17 inhibitor & Phase II & NCT03248531 & UCB Biopharma S.P.R.L. \\
\hline
\end{tabular}

IFX-1

IFX-1 is a first-in-class monoclonal antibody directed against complement factor C5a, which is one of the traditional activation products of the complement cascade. C5a is also involved in the activation of neutrophils and the production of proinflammatory cytokines, including TNF- $\alpha$. Systemic complement activation occurs in HS. In a recent study it was shown that $\mathrm{C}$ a level is significantly increased in the plasma of patients with HS comparing with healthy controls [83]. However, the negative correlation of circulating C5a concentration with HS severity was observed. Interestingly, C5a level in the plasma of HS sufferers was even greater than concentration reported for patients with severe sepsis or multiple organ failure [83]. IFX-1 by blocking C5a may be, therefore, helpful in regulating the inflammatory response in patients with $H S$.

In an open-label Phase II clinical trial the safety and efficacy of IFX-1 in HS patients were assessed [82,84]. 12 patients with Hurley Stage III HS were treated with IFX-1 at a dose regimen of $800 \mathrm{mg}$, administered intravenously on days $1,4,8,15,22,29,36,43$ and 50 . As a result, HiSCR score was obtained in a rate of $75 \%$ in patients at the end of the treatment period (day 50 ) and $83 \%$ after a 12 -week follow-up period (day 134). No adverse effects, allergic or anaphylactic reactions after drug infusion were reported during the course of the treatment.

In light of recent data, IFX-1 appears to be a new promising therapeutic approach for patients with HS who have failed to respond to previous conventional therapies or other biologicals. C5a blockade can become a new therapeutic option in diseases where increased systemic complement activation occurs, in particular HS.

A large randomized, double-blind, placebo-controlled, multicenter Phase II study on a group of 175 patients to estimate the efficacy and the safety of IFX-1 is currently under recruitment [45].

\section{Ongoing trials \& future perspective}

Despite the large progress, HS therapy often remains a serious challenge. There is still an unmet need for a new treatment options which can be achieved by range of potential targets directed against specific mechanisms. A new Phase II trial for HS management has begun in recent years using a inhibition of the targetable inflammatory pathways which are IL-1 $\alpha$, IL-17 and C5a. These cytokines also seem to be involved in HS pathogenesis, therefore their blockade appears as a new approach to therapy of the condition [45]. Currently several new biological preparations are being investigated, including MABp1, CJM112 and bimekizumab. The results failed with the drug MEDI8968 and the trial has been terminated early because of the lack of efficacy [45]. The investigational drugs for HS which are currently in clinical trials are presented in Table 2 [45].

With one approved biologic available, several drugs under investigation and the ongoing development of novel therapeutic agents that act in different specified pathways in the inflammatory cascade, the future of HS management looks promising. The era of targeted treatment will allow for a more 'personalized' approach directed against predictive biomarkers which dysregulation underlies HS pathomechanism. Certain therapies (currently under active investigation), including agents targeting IL-1 or IL-17 may occur as potentially promising options for HS therapy in the future. The current landscape of biologics promises continuous development of these preparations in the next few years with more innovative methods appearing on the market and offering new therapeutic approaches. Therefore, in the coming years, the final goal should be to improve the currently known preparations as well as search for new drugs and finally to find a balance among efficacy, toxicity and cost of therapy. Comparative studies including different preparations and dosing regimens of biologics would be particularly helpful to enhance their therapeutic effect.

\section{Conclusion}

Summarizing, conventional treatment options for HS have largely been disappointing and current systemic therapies for this condition still remain a serious challenge, though great progress has been made in HS management within recent years. A substantial therapeutic need still exists in HS because of its high prevalence and the burden it places on affected patients. 
Several cytokines have been found to drive inflammation in HS, including TNF- $\alpha$, IL-1 $\beta$, IL-17 and IL-23. Due to the role of immune dysregulation in HS pathogenesis, biologic therapy based on a targeted inhibition of these specific cytokines seems to create a promising option for patients with severe and moderate HS after conventional therapies proved insufficient.

According to current evidence, TNF- $\alpha$ inhibitors, especially ADA and IFX were found to be an effective and tolerable treatment modality for HS and appeared to significantly improve patients' QOL. Variable results have been seen with the use of other biologics in HS management, including ETA, ANA, UST, SEC and IFX-1. However, other agents still require more rigorous examination to be established as a therapeutic approach for this condition. Available data report usually good tolerance of biologics with mostly mild adverse events noticed. The results of the published studies on biologics in HS therapy are summarized in the Supplementary tables.

Up to date, ADA still remains as the only FDA/EMA-approved biologic drug in HS treatment and should be considered first, but other biologicals also play a increasing role in off-label therapy. Future large randomized controlled trials are needed to further establish the efficacy and safety profile of biologic agents in HS management.

\section{Executive summary}

\section{Background}

- Hidradenitis suppurativa (HS) is a chronic, debilitating dermatosis with occurrence of suppurative lesions, sinus tracks and scarring.

- Pathogenesis is multifactorial and a pilosebaceous unit occlusion, hyperkeratinization and bacterial superinfection play a key role.

- Several comorbid disorders (including immune-mediated inflammatory diseases), decreased quality of life and chronic pain appear in HS patients.

- Treatment of the condition is challenging- topical and systemic antibiotics, retinoids and hormone therapy are most commonly used, while biologics create a new promising option.

TNF- $\alpha$ inhibitors

- TNF- $\alpha$ seems to play a significant role in the pathogenesis of HS.

- Increased levels of TNF- $\alpha$ were found both in blood and skin lesions of patients suffering from HS.

- However, no significant difference was found in cytokine concentration before and after treatment with TNF- $\alpha$ inhibitors.

Adalimumab

- By blocking the biological activity of TNF- $\alpha$, adalimumab (ADA) regulates the innate immune response and affects the levels of other proinflammatory cytokines, including II-6, II-8, II-1 $\beta$ and sTNF-RI.

- The first reports about the efficacy of ADA on HS came from several case series and the effects were satisfactory but in a retrospective studies conducted by different authors, the results were contradictory due to ADA effectiveness.

- In randomized, double-blind, placebo-controlled trials, including one multicenter study, ADA appeared to be well tolerated and effective, especially when administered $40 \mathrm{mg}$ every week.

- $A D A$ is the only biologic agent approved by the and EMA for therapy of moderate to severe HS.

\section{Infliximab}

- Infliximab (IFX) is another monoclonal antibody that works against TNF- $\alpha$ and was found to decrease disease severity and improve patients' quality of life.

- In only one randomized, double-blind, placebo-controlled study conducted on a group of 38 patients, a significantly greater number of patients treated with IFX achieved at least $50 \%$ improvement in skin lesions compared with placebo.

- In the comparative study, IFX occurred to be more effective in all aspects than ADA with a mean $54 \%$ decrease in Sartorius score compared with baseline.

Etanercept

- Promising results regarding the efficacy of etanercept (ETA) (which is another TNF- $\alpha$ inhibitor) in HS therapy were obtained in several case series, as well as, in open cohort studies.

- However, the results were unsatisfactory in only one RCT trial and there was no significant difference in patient global assessment, physician global assessment or quality of life between ETA and placebo groups.

Anakinra

- Anakinra (ANA) is an IL-1 receptor antagonist which was originally registered for the treatment of moderate to severe rheumatoid arthritis.

- One author when assessing the efficacy of ANA in HS therapy in a group of 6 patients reported a clinically significant improvement of disease severity and a mean decrease of modified Sartorius Score in this study was 34.8 points. 
- In a larger randomized double-blind, placebo-controlled clinical trial 78\% of patients treated with ANA achieved a good clinical response which confirmed ANA as a potentially efficacious management of HS.

Ustekinumab

- Ustekinumab (UST) regulates specific components of the immune system by inhibiting (IL)-12 and IL-23, which were found to be increased in lesional skin of HS sufferers.

- According to results of several case reports including, in total, six patients, different outcomes were achieved from no treatment effect to complete response to UST therapy.

- In the only open-label clinical trial on UST efficacy, moderate to marked improvement of skin lesions (according to $\mathrm{mSs}$ ) was achieved in $82 \%$ and the Hidradenitis Suppurativa Clinical Response in $47 \%$ of patients.

Secukinumab

- Secukinumab (SEC) inhibits the inflammatory cascade by working against IL-17A, of which, expression was found to be significantly enhanced both in the blood and the skin of patients with $\mathrm{HS}$.

- Three cases have been published on SEC in HS treatment - the effects were satisfactory and all patients experienced a marked improvement in the course of the disease within a few months.

- Currently, SEC is being tested in a randomized placebo-controlled trial in a group of 21 patients.

IFX-1

- IFX-1 is an antibody directed against complement factor $\mathrm{C} 5 \mathrm{a}$, which is one of the activation products of the complement cascade and is notably increased in the plasma of HS patients.

- In an open-label Phase II clinical trial the efficacy of IFX-1 in HS patients was assessed and as a result Hidradenitis Suppurativa Clinical Response score was achieved in a rate of $75 \%$ in patients at day 50 and $83 \%$ at day 134 .

Ongoing trials \& future perspective

- Currently several new biological agents are being investigated in HS therapy, including MABp1, CJM112, bimekizumab and secukinumab.

- Novel biological agents targeted against specific elements of proinflammatory cascade, including IL-1 $\alpha$, IL-17 and C5a may occur as potentially promising options for HS therapy in the future.

Conclusion

- Several cytokines have been found to drive inflammation in HS, including TNF- $\alpha$, IL-1 $\beta$, IL-17 and IL-23 and their targeted inhibition appears to create a promising option for patients with severe and moderate HS after a failure of conventional therapies.

- TNF- $\alpha$ inhibitors, especially ADA and IFX were found to be effective and in general well tolerated therapy for HS and still more rigorous evaluation is needed for other agents

Financial \& competing interests disclosure

The authors have no relevant affiliations or financial involvement with any organization or entity with a financial interest in or financial conflict with the subject matter or materials discussed in the manuscript. This includes employment, consultancies, honoraria, stock ownership or options, expert testimony, grants or patents received or pending, or royalties.

No writing assistance was utilized in the production of this manuscript.

Supplementary data

To view the supplementary data that accompany this article please visit the journal website at: www.futuremedicine.com/doi/sup pl/10.2217/imt-2018-0090. References 85-121 refer to the supplementary tables.

\section{References}

Papers of special note have been highlighted as: $\bullet$ of interest; $\bullet \bullet$ of considerable interest

1. Jemec GB. Clinical practice. Hidradenitis suppurativa. N. Engl. J. Med. 366(2), 158-164 (2012).

2. Revuz J. Hidradenitis suppurativa. J. Eur. Acad. Dermatol. Venereol. 23(9), 985-998 (2009).

3. Sartorius K, Emtestam L, Jemec GB, Lapins J. Objective scoring of hidradenitis suppurativa reflecting the role of tobacco smoking and obesity. Br. J. Dermatol. 161(4), 831-839 (2009).

4. Vazquez BG, Alikhan A, Weaver AL, Wetter DA, Davis MD. Incidence of hidradenitis suppurativa and associated factors: a population-based study of Olmsted County, Minnesota. J. Invest. Dermatol. 133(1), 97-103 (2013).

5. Revuz JE, Canoui-Poitrine F, Wolkenstein $\mathrm{P}$ et al. Prevalence and factors associated with hidradenitis suppurativa: results from two case-control studies. J. Am. Acad. Dermatol. 59(4), 596-601 (2008).

6. Jemec GB, Heidenheim M, Nielsen NH. The prevalence of hidradenitis suppurativa and its potential precursor lesions. J. Am. Acad. Dermatol. 35(2 Pt 1), 191-194 (1996).

7. van der Zee $\mathrm{HH}$, van der Woude CJ, Florencia EF, Prens EP. Hidradenitis suppurativa and inflammatory bowel disease: are they associated? Results of a pilot study. Br. J. Dermatol. 162(1), 195-197 (2010). 
8. van der Zee HH, de Winter K, van der Woude CJ, Prens EP. The prevalence of hidradenitis suppurativa in 1093 patients with inflammatory bowel disease. Br. J. Dermatol. 171(3), 673-675 (2014).

9. Matusiak L, Szczech J, Kaaz K, Lelonek E, Szepietowski JC. Clinical characteristics of pruritus and pain in patients with hidradenitis suppurativa. Acta Derm. Venereol. 98(2), 191-194 (2018).

10. Matusiak L. Profound consequences of hidradenitis suppurativa: a review. Br. J. Dermatol. (2018). doi: 10.1111/bjd.16603. [Epub ahead of print]

11. Matusiak L, Bieniek A, Szepietowski JC. Hidradenitis suppurativa markedly decreases quality of life and professional activity. J. Am. Acad. Dermatol. 62(4), 706-708 (2010).

12. Wolkenstein P, Loundou A, Barrau K, Auquier P, Revuz J. Quality of life impairment in hidradenitis suppurativa. A study of 61 cases. J. Am. Acad. Dermatol. 56(4), 621-623 (2007).

13. Zouboulis CC, Desai N, Emtestam L et al. European S1 guideline for the treatment of hidradenitis suppurativa/acne inversa. J. Eur. Acad. Dermatol. Venereol. 29(4), 619-644 (2015).

-• Actual treatment algorithm for hidradenitis suppurativa (HS) recommended by the group of experts, including traditional therapies as well as biologic agents.

14. Ponikowska M, Matusiak L, Szepietowski JC. Current systemic treatment strategies for hidradenitis suppurativa. Expert Opin. Orphan Drugs. 5(3), 241-251 (2017).

15. Martinez F, Nos P, Benlloch S, Ponce J. Hidradenitis suppurativa and Crohn's disease: response to treatment with infliximab. Inflamm. Bowel Dis. 7(4), 323-326 (2001).

16. Matusiak L, Bieniek A, Szepietowski JC. Increased serum tumour necrosis factor-alpha in hidradenitis suppurativa patients: is there a basis for treatment with anti-tumour necrosis factor-alpha agents? Acta. Derm. Venereol. 89(6), 601-603 (2009).

- Reported elevated levels of tumor necrosis factor-alpha in a serum of HS patients provides a basis for treatment with TNF- $\alpha$ blockers.

17. van der Zee HH, de Ruiter L, van den Broecke DG et al. Elevated levels of tumour necrosis factor (TNF)- $\alpha$, interleukin (IL)-1 $\beta$ and IL-10 in hidradenitis suppurativa skin: a rationale for targeting TNF- $\alpha$ and IL-1 $\beta$. Br. J. Dermatol. 164(6), 1292-1298 (2011).

18. Mozeika E, Pilmane M, Nürnberg BM, Jemec GB. Tumour necrosis factor-alpha and matrix metalloproteinase-2 are expressed strongly in hidradenitis suppurativa. Acta. Derm. Venereol. 93(3), 301-304 (2013).

19. van der Zee HH, Laman JD, de Ruiter L, Dik WA, Prens EP. Adalimumab (antitumour necrosis factor- $\alpha$ ) treatment of hidradenitis suppurativa ameliorates skin inflammation: an in situ and ex vivo study. Br. J. Dermatol. 166(2), 298-305 (2012).

20. Jimenez-Gallo D, de la Varga-Martinez R, Ossorio-Garcia L, Collantes-Rodriguez C, Rodriguez C, Linares-Barrios M. Effects of adalimumab on T-helper-17 lymphocyte- and neutrophil-related inflammatory serum markers in patients with moderate-to-severe hidradenitis suppurativa. Cytokine 103, 20-24 (2018).

21. Samycia M, Brassard A. Adalimumab in treatment-resistant hidradenitis suppurativa following recurrence after extensive affected area excision: a review of biologics therapy. J. Cutan. Med. Surg. 17(1), 23-32 (2013).

22. Fotiadou C, Vakirlis E, Ioannides D. Spotlight on adalimumab in the treatment of active moderate-to-severe hidradenitis suppurativa. Clin. Cosmet. Investig. Dermatol. 19(9), 367-372 (2016).

23. Scheinfeld N. Treatment of coincident seronegative arthritis and hidradentis suppurativa with adalimumab. J. Am. Acad. Dermatol. 55(1), 163-164 (2006).

24. Harde V, Mrowietz U. Treatment of severe recalcitrant hidradenitis suppurativa with adalimumab. J. Dtsch. Dermatol. Ges. 7(2), 139-41 (2009).

25. Yamauchi PS, Mau N. Hidradenitis suppurativa managed with adalimumab. J. Drugs Dermatol. 8(2), 181-183 (2009).

26. Sotiriou E, Apalla Z, Vakirlis E, Ioannides D. Efficacy of adalimumab in recalcitrant hidradenitis suppurativa. Eur. J. Dermatol. 19(2), 180-181 (2009).

27. Gorovoy I, Berghoff A, Ferris L. Successful treatment of recalcitrant hidradenitis suppurativa with adalimumab. Case Rep. Dermatol. 1(1), 71-77 (2009).

28. Moul DK, Korman NJ. The cutting edge. Severe hidradenitis suppurativa treated with adalimumab. Arch. Dermatol. 142(9), 1110-1112 (2006).

29. Koilakou S, Karapiperis D, Tzathas C. A case of hidradenitis suppurativa refractory to anti-TNF- $\alpha$ therapy in a patient with Crohn's disease. Am. J. Gastroenterol. 105(1), 231-232 (2010).

30. Alzaga Fernandez AG, Demirci H, Darnley-Fisch DA, Steen DW. Interstitial keratitis secondary to severe hidradenitis suppurativa: a case report and literature review. Cornea 29(10), 1189-1191 (2010).

31. Reddick CL, Singh MN, Chalmers RJ. Successful treatment of superficial pyoderma gangrenosum associated with hidradenitis suppurativa with adalimumab. Dermatol. Online J. 16(8), 15 (2010).

32. Bosnić D, Zarković B, Beresić M, Zarkovic M, Anić B. Improvement of overlapping hidradenitis suppurativa and ankylosing spondylitis after the introduction of adalimumab. Reumatologia 54(6), 321-325 (2016). 
33. Bahillo Monné C, Honorato Guerra S, Schoendorff Ortega C, Gargallo Quintero AB. Management of hidradenitis suppurativa with biological therapy: report of four cases and review of the literature. Dermatology. 229(4), 279-287 (2014).

34. Blanco R, Martinez-Taboada VM, Villa I et al. Long-term successful adalimumab therapy in severe hidradenitis suppurativa. Arch. Dermatol. 145(5), 580-584 (2009).

35. Arenbergerova M, Gkalpakiotis S, Arenberger P. Effective long-term control of refractory hidradenitis suppurativa with adalimumab after failure of conventional therapy. Int. J. Dermatol. 49(12), 1445-1449 (2010).

36. Sotiriou E, Goussi C, Lallas A et al. A prospective open-label clinical trial of efficacy of every week administration of adalimumab in the treatment of hidradenitis suppurativa. J. Drugs Dermatol. 11(5), 15-20 (2012).

37. Amano M, Grant A, Kerdel FA. A prospective open-label clinical trial of adalimumab for the treatment of hidradenitis suppurativa. Int. J. Dermatol. 49(8), 950-955 (2010).

38. Miller I, Lynggaard CD, Lophaven S, Zachariae C, Dufour DN, Jemec GB. A double-blind placebo-controlled randomized trial of adalimumab in the treatment of hidradenitis suppurativa. Br. J. Dermatol. 165(2), 391-398 (2011).

- First reported double-blind randomized controlled trial (RCT) study for adalimumab (ADA) efficacy in HS therapy.

39. Kimball AB, Kerdel F, Adams D et al. Adalimumab for the treatment of moderate to severe hidradenitis suppurativa: a parallel randomized trial. Ann. Intern. Med. 157(12), 846-855 (2012).

- Two-phased RCT study on a large group of patients for ADA efficacy and safety.

40. Kimball AB, Okun MM, Williams DA et al. Two Phase 3 trials of adalimumab for hidradenitis suppurativa. N. Engl. J. Med. 375(5), 422-434 (2016).

-• Provides a registration studies for ADA as the first biologic agent in HS.

41. Zouboulis CC. Adalimumab for the treatment of hidradenitis suppurativa/acne inversa. Expert Rev. Clin. Immunol. 2(10), 1015-1026 (2016).

42. Delobeau M, Abdou A, Puzenat E et al. Observational case series on adalimumab-induced paradoxical hidradenitis suppurativa. J. Dermatolog. Treat. 27(3), 251-253 (2016).

43. Scheinfeld N, Sundaram M, Teixeira H, Gu Y, Okun M. Reduction in pain scores and improvement in depressive symptoms in patients with hidradenitis suppurativa treated with adalimumab in a Phase 2, randomized, placebo-controlled trial. Dermatol. Online J. 22(3), (2016).

44. Thomsen SF. A note on improvement in dermatology life quality index (DLQI) among patients with hidradenitis suppurativa treated with adalimumab. J. Dermatolog. Treat. 7, 1-2 (2018). doi: 10.1080/09546634.2018.1467537. [Epub ahead of print]

45. U.S. National Library of Medicine. https://clinicaltrials.gov

46. Paradela S, Rodríguez-Lojo R, Fernández-Torres R, Arévalo P, Fonseca E. Long-term efficacy of infliximab in hidradenitis suppurativa. J. Dermatolog. Treat. 23(4), 278-283 (2012).

47. Lesage $\mathrm{C}$, Adnot-Desanlis L, Perceau G et al. Efficacy and tolerance of prolonged infliximab treatment of moderate-to-severe forms of hidradenitis suppurativa. Eur. J. Dermatol. 22(5), 640-644 (2012).

48. Grant A, Gonzalez T, Montgomery MO, Cardenas V, Kerdel FA. Infliximab therapy for patients with moderate to severe hidradenitis suppurativa: a randomized, double-blind, placebo-controlled crossover trial. J. Am. Acad. Dermatol. 62(6), 205-217 (2010).

-. Only one conducted double-blind RCT study for infliximab efficacy, reporting a significant improvement comparing with placebo.

49. van Rappard DC, Leenarts MF, Meijerink-van't Oost L, Mekkes JR. Comparing treatment outcome of infliximab and adalimumab in patients with severe hidradenitis suppurativa. J. Dermatolog. Treat. 23(4), 284-289 (2011).

50. Adams DR, Yankura JA, Fogelberg AC, Anderson BE. Treatment of hidradenitis suppurativa with etanercept injection. Arch. Dermatol. 146(5), 501-504 (2010).

-• Only one reported randomized controlled trial for etanercept efficacy and safety.

51. Zangrilli A, Esposito M, Mio G, Mazzotta A, Chimenti S. Long-term efficacy of etanercept in hidradenitis suppurativa. J. Eur. Acad. Dermatol. Venereol. 22(10), 1260-1262 (2008).

52. Arriola-Villabos P, Díaz-Valle D, Alejandre-Alba N, López-Abad C, Méndez-Fernández R, Benítez-del-Castillo JM. Bilateral Candida chorioretinitis following etanercept treatment for hidradenitis suppurativa. Eye (Lond). 22(4), 599-600 (2008).

53. Henderson RL. Treatment of atypical hidradenitis suppurativa with the tumour necrosis factor receptor-Fc fusion protein etanercept. $J$. Drugs Dermatol. 5(10), 1010-1011 (2006).

54. Pelekanou A, Kanni T, Savva A et al. Long-term efficacy of etanercept in hidradenitis suppurativa: results from an open-label Phase II prospective trial. Exp. Dermatol. 19(6), 538-540 (2009).

55. Giamarellos-Bourboulis EJ, Pelekanou E, Antonopoulou A et al. An open-label Phase II study of the safety and efficacy of etanercept for the therapy of hidradenitis suppurativa. Br. J. Dermatol. 158(3), 567-572 (2008).

56. Cusack C, Buckley C. Etanercept: effective in the management of hidradenitis suppurativa. Br. J. Dermatol. 154(4), 726-729 (2006). 
57. Lee RA, Dommasch E, Treat J et al. A prospective clinical trial of open-label etanercept for the treatment of hidradenitis suppurativa. $J$. Am. Acad. Dermatol. 60(4), 565-573 (2009).

58. Sotiriou E, Apalla Z, Ioannidos D. Etanercept for the treatment of hidradenitis suppurativa. Acta. Derm. Venereol. 89(1), 82-83 (2009).

59. Lopez-Martin C, Tortajada Goitia B, Faus Felipe V, Gómez Sánchez A, Ferrer Soler F, Garrido Siles M. Partial response to etanercept in the treatment of hidradenitis suppurativa. Farm. Hosp. 35(4), 189.e1-189.e4 (2011).

60. Dinarello CA, van der Meer JWM. Treating inflammation by blocking interleukin-1 in humans. Semin. Immunol. 25(6), 469-484 (2013).

61. Pazyar N, Feily A, Yaghoobi R. An overview of interleukin-1 receptor antagonist, anakinra, in the treatment of cutaneous diseases. Curr. Clin. Pharmacol. 7(4), 271-275 (2012).

62. Leslie KS, Tripathi SV, Nguyen TV, Pauli M, Rosenblum MD. An open-label study of anakinra for the treatment of moderate to severe hidradenitis suppurativa. J. Am. Acad. Dermatol. 70(2), 243-251 (2014).

63. Tzanetakou V, Kanni T, Giatrakou S et al. Safety and Efficacy of Anakinra in Severe Hidradenitis Suppurativa: A Randomized Clinical Trial. JAMA Dermatol. 152(1), 52-59 (2015).

- Double-blind RCT study providing promising results on efficacy of anakinra in HS therapy.

64. Zarchi K, Dufour DN, Jemec GB. Successful treatment of severe hidradenitis suppurativa with anakinra. JAMA Dermatol. 149(10), 1192-1194 (2013).

65. Menis D, Maroñas-Jiménez L, Delgado-Marquez AM, Postigo-Llorente C, Vanaclocha-Sebastián F. Two cases of severe hidradenitis suppurativa with failure of anakinra therapy. Br. J. Dermatol. 172(3), 810-811 (2015).

66. van der Zee HH, Prens EP. Failure of anti-interleukin-1 therapy in severe hidradenitis suppurativa: a case report. Dermatology. 226(2), 97-100 (2013).

67. Russo V, Alikhan A. Failure of anakinra in a case of severe hidradenitis suppurativa. J. Drugs Dermatol. 15(6), 772-774 (2016).

68. Koutruba N, Emer J, Lebwohl M. Review of ustekinumab, an interleukin-12 and interleukin-23 inhibitor used for the treatment of plaque psoriasis. Ther. Clin. Risk Manag. 6, 123-141 (2010).

69. Benson JM, Peritt D, Scallon BJ et al. Discovery and mechanism of ustekinumab: a human monoclonal antibody targeting interleukin-12 and interleukin-23 for treatment of immune-mediated disorders. MAbs. 3(6), 535-545 (2011).

70. Schlapbach C, Hanni T, Yawalkar N, Hunger RE. Expression of the IL-23/Th17 pathway in lesions of hidradenitis suppurativa. J. Am. Acad. Dermatol. 65(4), 790-798 (2011).

71. Gulliver WP, Jemec GB, Baker KA. Experience with ustekinumab for the treatment of moderate to severe Hidradenitis suppurativa. J. Eur. Acad. Dermatol. Venereol. 26(7), 911-914 (2012).

72. Santos-Pérez MI, García-Rodicio S, Del Olmo-Revuelto MA, Pozo-Román T. Ustekinumab for hidradenitis suppurativa: a case report. Actas Dermosifiliogr. 105(7), 720-722 (2014).

73. Baerveldt EM, Kappen JH, Thio HB, van Laar JA, van Hagen PM, Prens EP. Successful long-term triple disease control by ustekinumab in a patient with Behcet's disease, psoriasis and hidradenitis suppurativa. Ann. Rheum. Dis. 72(4), 626-627 (2013).

74. Sharon VR, Garcia MS, Bagheri S et al. Management of recalcitrant hidradenitis suppurativa with ustekinumab. Acta Derm. Venereol. 92(3), 320-321 (2012).

75. Blok JL, Li K, Brodmerkel C, Horvátovich P, Jonkman MF, Horváth B. Ustekinumab in hidradenitis suppurativa: clinical results and a search for potential biomarkers in serum. Br. J. Dermatol. 174(4), 839-846 (2016).

76. Eisen DB. Ustekinumab, another biologic with potential to help patients with hidradenitis suppurativa? Br. J. Dermatol. 174(4), 718-719 (2016).

77. Matusiak L, Szczech J, Bieniek A, Nowicka-Suszko D, Szepietowski JC. Increased interleukin (IL)-17 serum levels in patients with hidradenitis suppurativa: implications for treatment with anti-IL-17 agents. J. Am. Acad. Dermatol. 76(4), 670-675 (2017).

78. Kelly G, Hughes R, Mc Garry T et al. Dysregulated cytokine expression in lesional and non-lesional skin in hidradenitis suppurativa. Br. J. Dermatol. 173(6), 1431-1439 (2015).

79. Thorlacius L, Theut Riis P, Jemec GBE. Severe hidradenitis suppurativa responding to treatment with secukinumab: a case report. Br. J. Dermatol. (2017). doi: 10.1111/bjd.15769. [Epub ahead of print]

80. Schuch A, Fischer T, Boehner A, Biedermann T, Volz T. Successful treatment of severe recalcitrant hidradenitis suppurativa with the interleukin-17A antibody secukinumab. Acta Derm. Venereol. 98(1), 151-152 (2017).

81. Jørgensen AHR, Yao Y, Thomsen SF. Therapeutic response to secukinumab in a 36-year-old woman with hidradenitis suppurativa. Case Rep. Dermatol. Med. 1-3 (2018).

82. Riis T, Thorlacius LR, Jemec GB. Investigational drugs in clinical trials for hidradenitis suppurativa. Expert Opin. Investig. Drugs. 27(1), 43-53 (2018).

83. Kanni T, Zenker O, Habel M, Riedemann N, Giamarellos-Bourboulis EJ. Complement activation in hidradenitis suppurativa: a new pathway of pathogenesis? Br. J. Dermatol. (2018). doi:10.1111/bjd.16428. [Epub ahead of print] 
84. Giamarellos E. Efficacy and safety of IFX-1, an anti-C5a monoclonal antibody in an open label Phase 2a study in patients with severe hidradenitis suppurativa (HS) not eligible for adalimumab. Presented at: 7th Conference of the European Hidradenitis Suppurativa Foundation. Rotterdam, The Netherlands, 7-9 February 2018.

85. Chinniah N, Cains GD. Moderate to severe hidradenitis suppurativa treated with biological therapies. Australas. J. Dermatol. 55(2), 128-131 (2014).

86. Martin-Ezquerra G, Masferrer E, Masferrer-Niubo M et al. Use of biological treatments in patients with Hidradenitis suppurativa. J. Eur. Acad. Dermatol. Venereol. 29(1), 56-60 (2015).

87. Van der Zee HH, Laman JD, de Ruiter L, Dik WA, Prens EP. Adalimumab (antitumour necrosis factor- $\alpha$ ) treatment of hidradenitis suppurativa ameliorates skin inflammation: an in situ and ex vivo study. Br. J. Dermatol. 166(2), 298-305 (2012).

88. Sullivan TP, Welsh E, Kerdel FA, Burdick AE, Kirsner RS. Infliximab for hidradenitis suppurativa. Br. J. Dermatol. 149(5), 1046-1049 (2003).

89. Fernandez-Vozmediano JM, Armario-Hita JC. Infliximab for the treatment of hidradenitis suppurativa. Dermatology. 215(1), 41-44 (2007).

90. Fardet L, Dupuy A, Kerob D et al. Infliximab for severe hidradenitis suppurativa: transient clinical efficacy in 7 consecutive patients. J. Am. Acad. Dermatol. 56(4), 624-628 (2007).

91. Brunasso AM, Delfino C, Massone C. Hidradenitis suppurativa: are tumour necrosis factor-alpha blockers the ultimate alternative? $\mathrm{Br}$. J. Dermatol. 159(3), 761-763 (2008).

92. van Rappard DC, Mooij JE, Baeten DL, Mekkes JR. New-onset polyarthritis during successful treatment of hidradenitis suppurativa with infliximab. Br. J. Dermatol. 165(1), 194-198 (2011).

93. Delage M, Samimi M, Atlan M et al. Efficacy of infliximab for hidradenitis suppurativa: assessment of clinical and biological inflammatory markers. Acta Derm. Venereol. 91(2), 169-171 (2011).

94. Van Rappard DC, Mekkes JR. Treatment of severe hidradenitis suppurativa with infliximab in combination with surgical interventions. Br. J. Dermatol. 167(1), 206-208 (2012).

95. Moriarty B, Jiyad Z, Creamer D. Four-weekly infliximab in the treatment of severe hidradenitis suppurativa. Br. J. Dermatol. 170(4), 986-987 (2014).

96. Pedraz J, Dauden E, Perez-Gala S, Goiriz-Valdes R, Fernandez-Penas P, Garcia-Diez A. Hidradenitis suppurativa. Response to treatment with infliximab. Actas Dermosifiliogr. 98(5), 325-331 (2007).

97. Mekkes JR, Bos JD. Long-term efficacy of a single course of infliximab in hidradenitis suppurativa. Br. J. Dermatol. 158(2), 370-374 (2008).

98. Katsanos KH, Christodoulou DK, Tsianos EV. Axillary hidradenitis suppurativa successfully treated with infliximab in a Crohn's disease patient. Am. J. Gastroenterol. 97(8), 2155-2156 (2002).

99. Adams DR, Gordon KB, Devenyi AG, Ioffreda MD. Severe hidradenitis suppurativa treated with infliximab infusion. Arch.Dermatol. 139(12), 1540-1542 (2003).

100. Lebwohl B, Sapadin AN. Infliximab for the treatment of hidradenitis suppurativa. J. Am. Acad. Dermatol. 49(5), $275-276$ (2003).

101. Roussomoustakaki M, Dimoulios P, Chatzicostas C et al. Hidradenitis suppurativa associated with Crohn's disease and spondyloarthropathy: response to anti-TNF therapy. J. Gastroenterol. 38(10), 1000-1004 (2003).

102. Rosi YL, Lowe L, Kang S. Treatment of hidradenitis suppurativa with infliximab in a patient with Crohn's disease. J. Dermatolog. Treat. 16(1), 58-61 (2005).

103. Thielen AM, Barde C, Saurat JH. Long-term infliximab for severe hidradenitis suppurativa. Br. J. Dermatol. 155(5), 1105-1107 (2006).

104. Moschella SL. Is there a role for infliximab in the current therapy of hidradenitis suppurativa? A report of three treated cases. Int. J. Dermatol. 46(12), 1287-1291 (2007).

105. Kwan CK, Chong LY. A Chinese man with chronic recalcitrant hidradenitis suppurativa successfully treated with infliximab. Hong Kong J. Dermatol. Venereol. 16, 206-210 (2008).

106. Pedraz J, Penas PF, Garcia-Diez A. Pachyonychia congenita and hidradenitis suppurativa: no response to infliximab therapy. J. Eur. Acad. Dermatol. Venereol. 22(12), 1500-1501 (2008).

107. Antonucci A, Negosanti M, Negosanti L, Iozzo I, Varotti C. Acne inversa treated with infliximab: different outcomes in 2 patients. Acta Derm. Venereol. 88(3), 274-275 (2008).

108. Elkjaer M, Dinesen L, Benazzato L, Rodrigues J, Logager V, Munkholm P. Efficacy of Infliximab treatment in patients with severe Fistulizing Hidradenitis Suppurativa. J. Crohns Colitis 2(3), 241-245 (2008).

109. Montes-Romero JA, Callejas-Rubio JL, Sanchez-Cano D, Gonzalez-Martinez FJ, Navas-Parejo A, Ortego-Centeno N. Amyloidosis secondary to hidradenitis suppurativa. Exceptional response to infliximab. Eur. J. Intern. Med. 19(6), 32-33 (2008).

110. Lozeron P, Denier C, Lacroix C, Adams D. Long-term course of demyelinating neuropathies occurring during tumor necrosis factor- $\alpha$ blocker therapy. Arch. Neurol. 66(4), 490-497 (2009). 
111. Poulin Y. Successful treatment of hidradenitis suppurativa with infliximab in a patient who failed to respond to etanercept. J. Cutan. Med. Surg. 13(4), 221-225 (2009).

112. Obadia DL, Daxbacher EL, Jeunon T, Gripp AC. Hidradenitis suppurativa treated with infliximab. An. Bras. Dermatol. 84(6), 695-697 (2009).

113. Goertz RS, Konturek PC, Naegel A et al. Experiences with a longterm treatment of a massive gluteal acne inversa with infliximab in Crohn's disease. Med. Sci. Monit. 15(1), 14-18 (2009).

114. García-Rabasco AE, Esteve-Martínez A, Zaragoza-Ninet V, Sánchez-Carazo JL, Alegre-de-Miquel V. Pyoderma gangrenosum associated with hidradenitis suppurativa: a case report and review of the literature. Actas Dermosifiliogr. 101(8), 717-721 (2010).

115. Alecsandru D, Padilla B, Izquierdo JA, Fernandez-Cruz E, Sanchez-Ramon S. Severe refractory hidradenitis suppurativa in an HIV-positive patient successfully treated with infliximab. Arch. Dermatol. 146(12), 1343-1345 (2010).

116. Torres T, Selores M. Treatment of hidradenitis suppurativa with infliximab. An. Bras. Dermatol. 85(4), 576 (2010).

117. Lasocki A, Sinclair R, Foley P, Saunders H. Hidradenitis suppurativa responding to treatment with infliximab. Australas. J. Dermatol. 51(3), 186-190 (2010).

118. De Souza A, Solomon GE, Strober BE. SAPHO syndrome associated with hidradenitis suppurativa successfully treated with infiximab and methotrexate. Bull NYU Hosp. Jt. Dis. 69(2), 185-187 (2011).

119. Husein-ElAhmed H, Fernandez-Pugnaire MA, Ruiz-Carrascosa JC. Severe hidradenitis suppurative in an HIV-positive male: use of multiple treatment modalities, including tumor necrosis factor blockade. AIDS Patient Care STDS. 25(9), 507-508 (2011).

120. von Preussen AC, Flux K, Hartschuh W, Hartmann M. Acne inversa successfully treated with infliximab. Int. J. Dermatol. 51(8), 1011-1013 (2012).

121. dos Santos CH, Netto PO, Kawaguchi KY, Parreira Alves JA, de Alencar Souza VP, Reverdito S. Association and management of Crohn's disease plus hidradenitis suppurativa. Inflamm. Bowel Dis. 18(4), 801-802 (2012). 
